\title{
Identification and assessment of a nonlinear dynamic actuator model for controlling an experimental flexible wing
}

\author{
Martin Tang ${ }^{1} \cdot$ Marc Böswald $^{1} \cdot$ Yves Govers ${ }^{1} \cdot$ Manuel Pusch $^{2}$
}

Received: 12 February 2020 / Revised: 12 February 2021 / Accepted: 31 March 2021 / Published online: 16 April 2021

(c) The Author(s) 2021

\begin{abstract}
In this paper, the effect of nonlinear actuator dynamics on the performance of an active load alleviation system for an experimental flexible wing is studied. Common nonlinearities such as backlash or rate limits are considered for the control surface actuator. An aeroelastic simulation model of a flexible wing with control surface is being used. With this, a parameter study is carried out to quantify the impact of the individual nonlinearities on the overall closed-loop performance by means of describing functions. Finally, the nonlinear actuator model with parameters identified from dedicated tests is experimentally validated allowing for an accurate prediction of the expected gust load alleviation performance.
\end{abstract}

Keywords System identification · Active load alleviation · Wind tunnel experiment · Actuator modeling · Nonlinear dynamics $\cdot$ Aeroservoelasticity

\section{Introduction}

The design of future aircraft shall enable a reduction in fuel consumption and operating cost. Active load alleviation allows for notable structural mass savings, which will in turn lead to decreased fuel consumption and, thus, lower operating costs and less emissions. The German Aerospace Center (DLR) has investigated the benefits of an active load alleviation system by numerical simulations and by wind tunnel experiments within the internal research activity "KonTeKst" [1-4]. For the design of a suitable control law, a numerical model of the open-loop system is required. The system considered here involves aerodynamics, structural

Martin Tang

martin.tang@dlr.de

Marc Böswald

marc.boeswald@dlr.de

Yves Govers

yves.govers@dlr.de

Manuel Pusch

manuel.pusch@dlr.de

1 German Aerospace Center (DLR), Institute of Aeroelasticity, Bunsenstr. 10, 37073 Göttingen, Germany

2 German Aerospace Center (DLR), Institute of System Dynamics and Control, Münchener Str. 20, 82234 Weßling, Germany dynamics, and actuator dynamics. This work focuses on the dynamics of the actuator used in the wind tunnel model. More precisely, on the effect of nonlinearities in the actuation system on the performance of a gust load alleviation system tested on an experimental flexible wing depicted in Fig. 1.

The open-loop system is an aeroelastic system with elastic, inertial and aerodynamic forces. Their schematic interaction is seen in the block diagram in Fig. 2, introduced by Fung [5]. When considering active load alleviation, a controller (dashed line) and an actuator (dash-dotted line) have to be added to the system, to represent the dynamics appropriately. Obviously, the controller performance largely depends on the performance of the flap actuation system, which often features nonlinearities. Typical nonlinearities are backlash, deflection limits (i.e., saturation in deflection), rate limits (i.e., saturation in velocity) and acceleration limits (i.e., saturation in acceleration) [6]. For control law design and gust response analysis, however, it is state-of-the-art that these nonlinearities are neglected and linearized models are used instead. In this case, the aforementioned nonlinearities are taken into account by retaining sufficiently high robustness margins $[7,8]$.

In general, numerous concepts for flap actuation in wind tunnel models exist; for instance, piezo stack actuators which are directly integrated into the wing structure and feature a high bandwidth. Nevertheless, high-end amplifiers are 


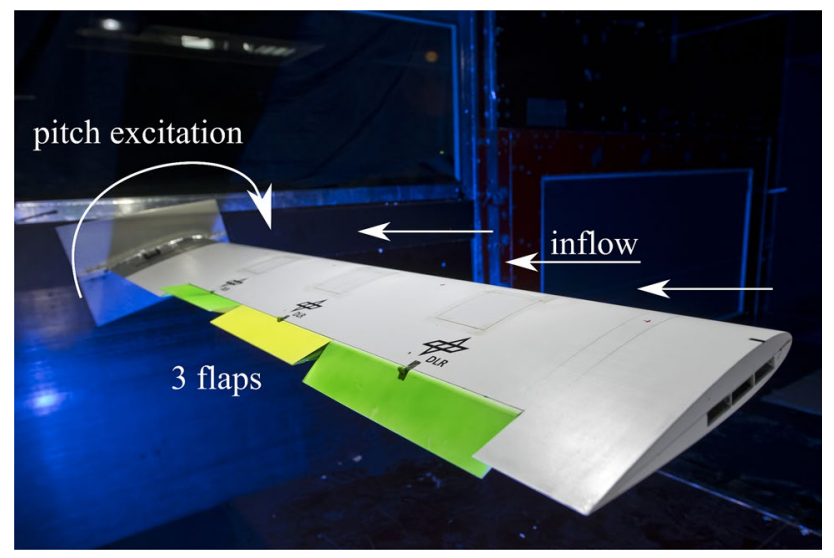

Fig. 1 Experimental setup of flexible wing with control surfaces mounted in the wind tunnel

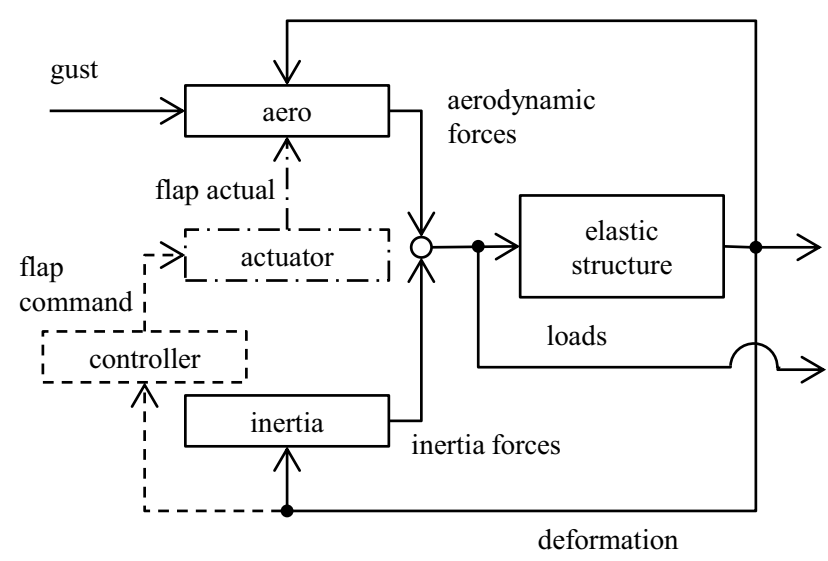

Fig. 2 Block diagram of aeroservoelastic systems [5]

required and travel range is limited [9]. Flaps may also be driven directly at the hinge line. This saves some mechanical linkage and generally reduces potential backlash within the actuation system. However, sufficient installation space must be available at the hinge line to include the direct drive. For RC planes, state-of-the-art actuation concepts comprise a servo and linkage. The servo can be placed within the wing at a suitable location with enough space for installation and cable routing. A linkage is required, typically consisting of a bell crank at the servo output shaft, a bell crank at the flap hinge, and a rod connecting the two bell cranks. Such a mechanical setup typically introduces additional backlash, e.g., from the summation of the freeplay in mechanical joints. In the considered flexible wind tunnel model of a wing with flap, servos have been chosen as primary actuation elements.

The design and manufacturing of the wing structure within this project has been presented earlier [2]. Numerical models of the mechanical structure as well as the deformation induced unsteady aerodynamic forces are built up within the design process. To further model the overall actuation system, detailed models of its components and their interaction are required [10]. In general, such mathematical models are not available, especially not for low-cost servos as used in this project. The considered actuator is, thus, treated as a black box and an equivalent simulation model is determined experimentally. Nominal data of the servos, such as maximum torque or maximum rotation speed of the output shaft, are given by the manufacturer. But the overall dynamics can only be identified after physical assembly of all subsystems.

Nonlinearities in actuators have been extensively studied for hydraulic actuators of full-scale aircraft for flight control. Taylor et al. [11] assessed the impact of nonlinear actuator dynamics on flight control performance. Fielding and Flux [6] give an overview of common nonlinearities in hydraulic systems and analyze them with appropriate methods. Also, the modeling of hydraulic actuators is described in their work. Stirling and Cowling [12] modeled a hydraulic actuator for a combat aircraft and simulated the nonlinear behavior of the actuation system. Banavara and Newsom [13] studied the effect of a nonlinear actuator on a full-scale aircraft. In their paper, the open- and closed-loop system behavior is studied in time domain using a linear model of the aerodynamics and structural dynamics with an additional nonlinear actuator model. Klyde et al. [14] predicted pilot induced oscillation due to actuator rate limits. To the knowledge of the authors, nonlinearities in actuators have not been studied for small scale electric servos applied to gust load alleviation. Nevertheless, the type of nonlinearities given in the literature for hydraulic actuators are also observed in electric servo motors. For example, servo motors also show backlash and run into limits like maximum deflection or rate, although these properties of hydraulic and electric systems differ significantly in their values. Thus, the same methodologies can be used to study and simulate electric actuation systems.

In the flight control domain, the rigid body properties of the aircraft are of primary importance. Control surface are located at positions which allow for efficient control of the rigid body pitch, roll and yaw motion. It is not desired to excite flexible modes with the actuation system. Nevertheless, sensors such as inertia platforms used for observing the actual state of the system, also detect flexible dynamic deformations at higher frequencies. If these sensor signals are fed back through a controller to the actuators, it can lead to an undesired or even unstable behavior when these signals fall within the operating bandwidth of the actuators [15, 16]. This has become a major challenge in modern aircraft designs, where a better aircraft performance is achieved at the cost of an increased coupling of rigid-body and flexible dynamics $[17,18]$. Within the "KonTeKst" project, the focus lies on active damping of flexible modes with the goal of reducing structural loads during gust encounter. Hence, 
electric actuators of sufficient bandwidth are required in contrast to actuators used for flight control.

Regarding parameter estimation, Schallert et al. [19] propose a method for automated backlash measurement. Cologni et al. [20] created a nonlinear model for electrohydraulic actuator and identified the needed parameters, whereas Ling et al. [21] used a black box model and identified parameters in order to create a mathematical model reproducing the same outputs. Regan [22] tested several electric servos in an extensive study for application in active flutter suppression. Amplitude-dependent system behavior was detected, which indicates nonlinear actuator dynamics.

From the above literature survey, it is seen that hydraulic actuators for real aircraft have been primarily assessed for the development of flight control laws, whereas the interest of this paper lies in electric servos for active load alleviation systems. Methodologies applied for parameter identification of nonlinear hydraulic actuators are utilized here to identify the parameters of a nonlinear substitute model for the dynamics of an electric flap actuator.

The present paper is organized in four sections and a list of references. Section 2 presents the modeling of the flap actuators and the aeroelastic system itself. Typical actuator nonlinearities are discussed there. The actuator is described as a first-order system augmented by nonlinear dynamics. The closed-loop performance of the aeroelastic system is simulated to assess the effect of the nonlinear actuator dynamics on the overall performance. In Sect. 3, the actuator design is described as well as the test bed, used to identify the dynamics of the flap actuator. The identification process for nonlinear parameters is discussed in detail. Measured data are compared with simulated data obtained with the identified parameters for the nonlinear actuator model. Finally, controller performance losses due to the identified nonlinearities are estimated. The conclusions are presented in Sect. 4, followed by a list of references.

\section{Simulation}

In this section, common nonlinearities occurring in actuation systems are studied based on numerical simulations. To that end, different nonlinearities are first reviewed and their effect on the input-output behavior of a single actuator is described. Then, the models for aerodynamics and structural dynamics are briefly introduced. Subsequently, a sensitivity study is carried out to quantify the impact of the considered nonlinearities on controller performance.

\subsection{Nonlinearities in actuation systems}

We first discuss nonlinearities occurring in actuation systems. Typically, limitations in deflection, rate and acceleration as well as backlash in the mechanical integration are seen. Due to thermal expansion during operation, backlash is needed. Nevertheless, it should be kept as low as possible. It is known that backlash usually reduces controller performance and can cause limit cycle oscillations [13, 23 ] or even instabilities. If commanded deflection is relatively low in comparison to the backlash, high attenuation with significant phase shift is seen. If the backlash is small in comparison to commanded deflection, linear behavior is observed.

Apart from backlash, the mechanical design of the actuator limits its maximum deflection. If the control law demands more deflection, the flap will collide with another part. As a safety measure, the commanded deflection is typically restricted to a certain minimum and maximum value. This type of nonlinearity does not introduce a phase lag but decreases the amplitude in case of saturation.

The bearings in the actuator limit the achievable velocity, also called rate. Note that actuator velocity may also be limited intentionally to reach a desired operating life time. This limitation is also called rate limit. If the demand exceeds the rate limit, the actual deflection starts to lag behind and the maximum amplitude is not reached anymore. Large velocities may result from large amplitudes or large frequencies in the command signal. Hence, this nonlinearity is also frequency dependent.

Also, servo motors cannot apply more than the maximum torque, which also limits the maximum acceleration of the control surface. If the demanded acceleration is too high, the actuator is not able to follow, which leads to amplitude attenuation and phase lag. The effect of this nonlinearity is seen if the commanded acceleration requires more torque than the maximum torque of the servo. The characteristics of this type of nonlinearity are quite similar to rate limit, also frequency dependent.

Furthermore, dead time has been observed. Internal electronics introduces delay while processing digitized signals. This nonlinearity does not affect amplitude but only phase, since the output signal is delayed by a certain time.

Besides, other nonlinearities in actuation systems have been reported, which may considerably affect the performance on the control loop. Friction in actuation systems cannot be avoided. Nevertheless, occurring forces in actuation systems are commonly not modeled in detail due to their negligible effect. Another nonlinear behavior is jump resonance. The frequency response function jumps at a certain excitation frequency to higher amplitudes, but this type of nonlinearity has not been observed within this test.

\subsection{Describing function}

For linear analysis, gain and phase are commonly used to describe dynamic systems. This concept is extended for the 
nonlinear case with describing functions. However, the gain and phase are amplitude dependent. If a linear system is excited by a single sine signal, it responds with a sine signal at the excitation frequency. In contrast, nonlinear systems may also respond with integer multiples of the fundamental sine excitation. Also, an amplitude-dependent behavior can be observed. In describing function analysis, response is reduced to the fundamental harmonic

$a=\int_{0}^{T} y e^{j \omega t} \mathrm{~d} t$

where $a$ is the complex response reduced to the fundamental frequency, $\omega$ is the frequency of excitation, and $y$ is the response of the system. Repeating this analysis for different excitation amplitudes allows then the description of amplitude-dependent behavior. Herein, this analysis is used to describe the amplitude gain and phase shift of chosen nonlinearities on the response for single stationary sine excitation.

As an illustrative example, the describing function for backlash is presented in the following. Figure 3 shows the result for a commanded sine signal of $1^{\circ}$ amplitude and an actuator backlash of $1^{\circ}$. The dash-dotted line shows the commanded deflection and the solid line shows the actual deflection. The actual deflection starts to revert its direction when the commanded signal passes the $1^{\circ}$ backlash. Due to the backlash nonlinearity, a phase shift is introduced and also the amplitude is attenuated by half the backlash. The dashed curve shows an approximation of the response with a single sine curve according to Eq. (1).

The aforementioned procedure is repeated for several excitation amplitudes, such that the approximated amplitude is given as function of the excitation amplitude, shown in Fig. 4. If the commanded amplitude increases, the ratio between backlash and amplitude of the command signal decreases and the influence of the backlash is reduced. Up to half the backlash, the actuator is not following the command at all. Then, the actuator starts to move but the amplitude is attenuated and the phase shift is significant. With increasing amplitudes, the attenuation is negligible and the phase

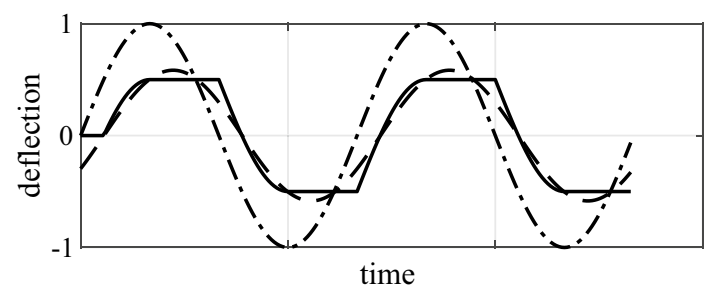

Fig. 3 Effect of backlash on the actuator; dash-dotted: commanded deflection, solid: actual deflection, dashed: approximated actual deflection as sine curve
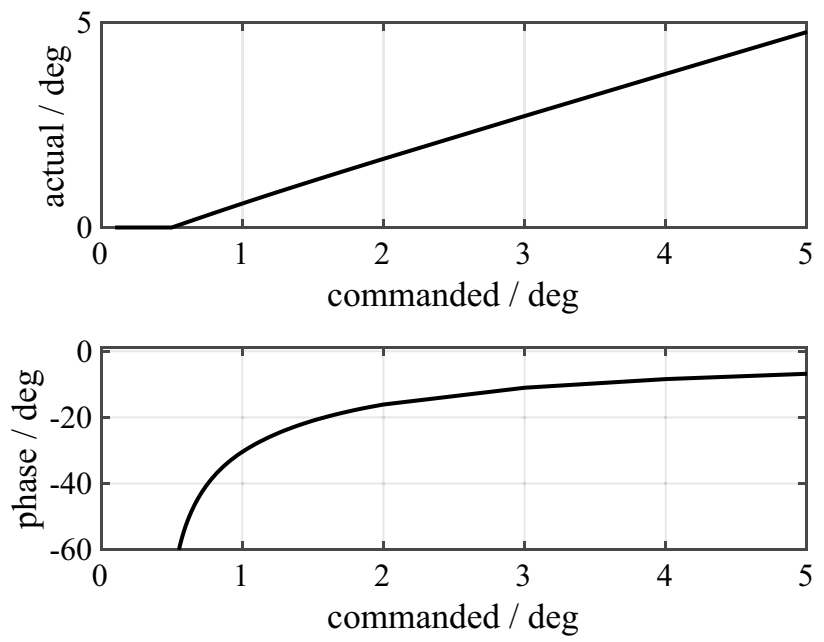

Fig. 4 Describing function for backlash nonlinearity

shift is reduced as well. As one can see, backlash results in a constant phase shift and constant amplitude attenuation.

More details on describing function analysis of actuator nonlinearities are discussed by Fielding and Flux [6] or Ackermann and Bünte [24]. Describing functions for the aforementioned nonlinearities are presented by Tang et al. [25].

\subsection{Integrated simulation model}

The integrated simulation model used herein consists of an aeroelastic model of the flexible wing augmented with nonlinear actuator models and a gust load alleviation system. A detailed description of the individual model parts is given by Pusch et al. [26] and summarized as follows.

\subsubsection{Flexible wing}

The considered experimental flexible wing features a span of $1.6 \mathrm{~m}$, a chord length of $0.25 \mathrm{~m}$, and a symmetric NACA0015 airfoil. The structural dynamics of the wing are modeled based on a finite element (FE) model which is directly obtained from the aeroelastic-tailoring process used to design the wing [27-29]. The structural layout comprises load carrying composite skins and a foam core, represented in the FE model as shell and volume elements, respectively, as depicted in Fig. 5. The flaps were modeled as beams. Point mass representations of all non-structural parts were used to achieve a realistic mass representation. The FE model is condensed and a modal analysis is carried out, where only the first eight flexible modes are kept while the remaining higher-frequent modes are truncated. Furthermore, the rigid-body dynamics are constrained to a pitching motion around the quarter-chord line as the wing is 


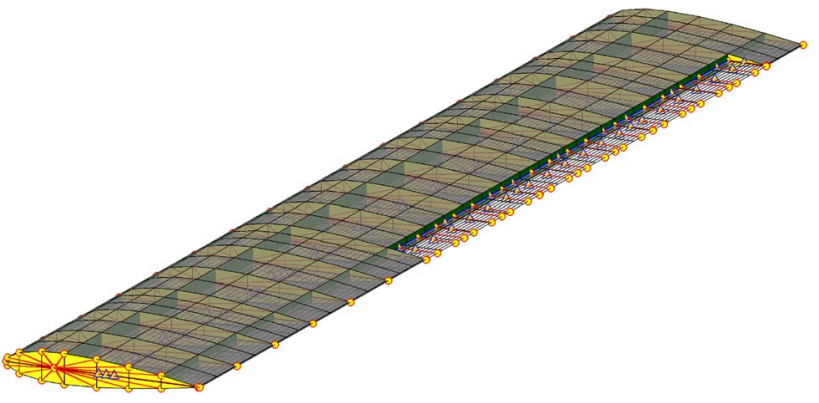

Fig. 5 FEM model of wing structure

mounted on a pitch excitation system at its root for simulating gust excitations.

The aerodynamic model is obtained in frequency domain using the doublet lattice method (DLM) [30], which captures also unsteady aerodynamic effects. To that end, the lifting surfaces are discretized by aerodynamic panels, where the panel size of the trailing edge flaps is reduced for a higher modeling accuracy. In order to allow for nonlinear time domain simulations, a rational function approximation (RFA) according to Roger [31] is carried out. The resulting unsteady aerodynamic model is reduced to an order of 20 by means of balanced truncation [32] and depends on the velocity of the air flow and its density.

Eventually, the structural dynamics model and the unsteady aerodynamic model are coupled yielding an aeroelastic model of the experimental flexible wing. Note that the aeroelastic model is determined for fixed velocities and represented as a linear time-invariant system. More details on the used aeroelastic modeling procedure are given by Kier and Looye [33].

\subsubsection{Gust load alleviation system}

For active gust load alleviation, the experimental wing is equipped with 8 vertical acceleration sensors and three trailing edge flaps with a size of $30 \mathrm{~cm}$ (span-wise) by $5 \mathrm{~cm}$ (chord-wise). The control law is designed using the approach described by Pusch et al. [34], which suggests an $\mathcal{H}_{2}$-optimal blending of control inputs and measurement outputs. In doing so, the loads-dominating first wing bending mode can be effectively isolated and subsequently damped by a simple single-input single-output (SISO) controller. The resulting controller structure in interconnection with the plant is depicted in Fig. 6.

\subsubsection{Flap actuators}

It is preferable to have a detailed model of the servo motor, where all components are described with mathematical equations for better insight into the actuator. But since the

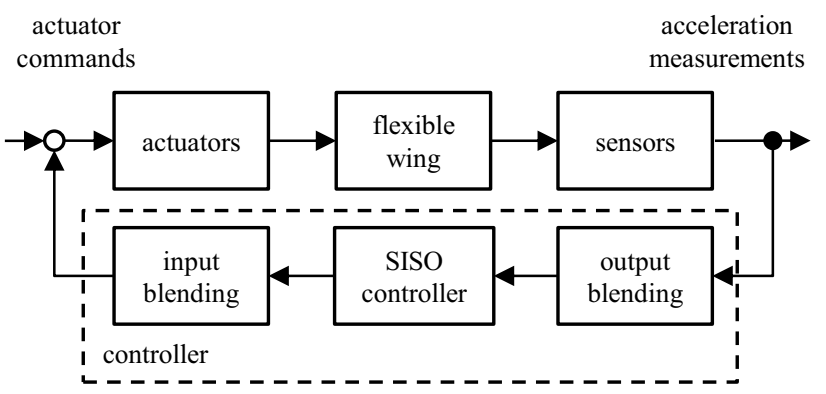

Fig. 6 Closed-loop interconnection

internal structure of the servo is unknown, especially the internal electronics and the position tracking controller, it is treated as black box system. Eventually, the actuation system is modeled as a first-order system extended by the nonlinear behavior described in Sect. 2.1. Also, higher-order systems have been investigated, but with no benefit. So, a first-order system is chosen. The structure of the model is shown in Fig. 7. The commanded flap deflection is used as input. First, the input is delayed by dead time which represents the internal data processing of the servo. Then, the signal is forwarded to a first-order system such that the actual angular velocity is generated. Since the servo is generating a torque first to turn the shaft, the acceleration limit is active first. Thus, an acceleration limit is chosen first. Then, rate is limited if the servo runs into power limit. After that, the signal is integrated in time and backlash as well as deflection limit is applied onto it. Backlash is chosen first, because it is assumed that mechanical connections with the shaft of the servo are causing backlash. Mechanical properties of the flap design leads then to deflection limits. Finally, the derivative of the signal is computed and put out as flap velocity. This input and output relation is chosen for the later integration into the whole model of the flexible wing. It is noteworthy that different orders of those nonlinear blocks might differ in overall dynamic behavior of this system.

In this setup, two sources of dead time exist. First, the real-time controller which is used for the loads alleviation runs with a sample rate of $1 \mathrm{kHz}$ (i.e., outer control loop), and second, the internal position tracking controller of the servo (i.e., inner control loop), which is considered a black box. Dead time cannot be represented as a linear differential

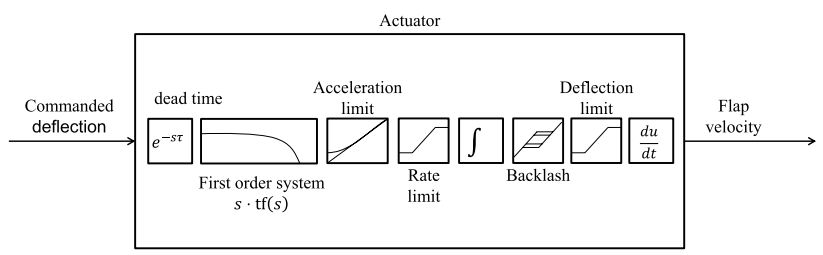

Fig. 7 Structure of the nonlinear actuator model 
equation; hence, it is often neglected in analysis but considered in the phase margin requirements within control design.

\subsection{Simulations of actuator only}

The nonlinear actuator model shown in Fig. 7 is implemented in Simulink and for each block the identified parameters introduced in the next section are used accordingly. To characterize its system behavior, a non-parametric identification has been carried out. Transfer functions at different deflection angles using sine sweeps are computed. This signal is similar to stationary sine signals, used for computing describing functions but are faster in application if a frequency range is of interest. A sine sweep is given as input to the simulation model and flap deflection is measured as output. With these two signals, a transfer function is computed for sine sweeps at different excitation level.

Figure 8 depicts the simulated transfer functions. Commanded deflection angles were $3^{\circ}$ (dotted line), $5^{\circ}$ (dashdotted line), $10^{\circ}$ (dashed line), $20^{\circ}$ (solid line). Looking at the phase diagram up to $10 \mathrm{~Hz}$, it is seen that dead time introduces a linear phase lag. Having a look onto the gain diagram, it is seen that gain increases with increasing amplitude level of the commanded deflection. Ideally, gain is one at all frequencies. The reason is free play and the explanation is seen in Fig. 4. The higher the commanded deflection in relation to free play, the actual deflection gets closer. Also an offset between different amplitude levels are seen in the phase signal, which is also represented in Fig. 4. Another phenomenon seen in Fig. 8 is the varying roll-off frequency for each amplitude level. This can be explained by either acceleration or rate limitations. The velocity and also acceleration are increasing with higher frequencies, but also with higher amplitudes. So limitations are reached at
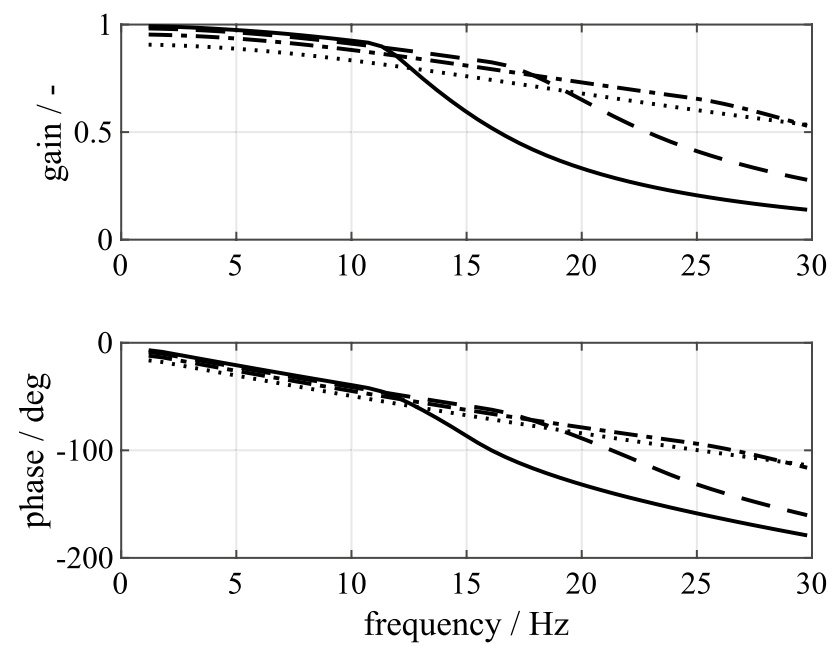

Fig. 8 Simulated transfer functions for actuator model. Dotted: $3^{\circ}$, dash-dotted: $5^{\circ}$, dashed: $10^{\circ}$, solid: $20^{\circ}$ lower frequencies for higher amplitudes, which results in gain and phase loss.

\subsection{Closed-loop simulations}

For the closed-loop simulations, the integrated simulation model described in Sect. 2.3 is used, where the wind speed is fixed at $40 \mathrm{~m} / \mathrm{s}$. Furthermore, all three actuators are assumed to be identical and have the same model properties at all time.

The describing function method is utilized to describe the behavior of the nonlinear system, according to Sect. 2.1. A sine signal at $8 \mathrm{~Hz}$ with an amplitude of $1^{\circ}$ is chosen as pitch excitation. This frequency is close to the first structural mode, such that the structure responds with high amplitudes. Thus, flap deflections commanded by the controller are expected to be high as well. Clearly, the resulting root bending moment is also harmonic, due to sine excitation. As discussed earlier, nonlinear systems might respond with multiple harmonics. Only the fundamental harmonic of the wing root bending moment is considered and the phase shift from wing root bending moment is given with respect to the pitch excitation. In the beginning, all nonlinearities are ignored to compute a reference response of the system. Afterwards, each nonlinearity is studied individually by variation of the corresponding nonlinear parameter, while all other parameters remain constant. In this way, the effects of the individual nonlinearities on the controller performance can be investigated separately. The results are compared to the reference case without any nonlinearity. The performance $P$ of the controller is defined as ratio of bending moment in openloop and closed-loop configuration, as following

$P=\frac{M_{\mathrm{OL}}-M_{\mathrm{i}}}{M_{\mathrm{OL}}-M_{\mathrm{CL}}}$,

where $M_{\mathrm{OL}}$ is the root bending moment of the system without load alleviation and $M_{\mathrm{CL}}$ is the root bending moment of the system with activated load alleviation system. $M_{\mathrm{i}}$ is the current root bending moment for a specified nonlinear behavior. In conclusion, the performance $P$ equals 1 for the linear reference case and degrades to 0 if no loads alleviation is achieved. The reference loads reduction is $16.4 \mathrm{~N} \mathrm{~m}$ and is represented in the denominator.

In simulations, it is possible to activate one single nonlinearity while others stay perfectly linear. This way, the impact of each nonlinearity is estimated individually.

\subsubsection{Backlash}

First, the backlash parameter is investigated by variation from $0^{\circ}$ to $4^{\circ}$. Although $1^{\circ}$ is already more than $50 \%$ of the reference deflection, the performance loss is only $16 \%$. 
Increasing backlash to $4^{\circ}$, controller performance loss is around $83 \%$. Figure 9 depicts the described behavior. Backlash is normalized with respect to the maximum reference deflection of $1.7^{\circ}$. It is noteworthy that the backlash parameter is varied up to double the reference deflection. However, the effect is rather small since the load alleviation controller is able to compensate the backlash. If control commands remain inside the backlash area, such that the flaps are not moving, the command signal is increased by the controller with deflections, outside the backlash area, hence loads are reduced again. In conclusion, this results in loads reduction although the backlash parameter is set to a higher value than the reference deflection, i.e., normalized backlash greater than 1. Still, this compensation also has limits if the backlash is too large due to the phase loss with increasing backlash, as seen in Fig. 4 and also in Fig. 9.

\subsubsection{Deflection limit}

Subsequently, the impact of deflection limit nonlinearity is studied by limiting maximum deflection of the flaps. The geometric constraint from wing design allows $\pm 10^{\circ}$ flap deflection. Under normal conditions, this limit is not reached. This means, that deflection commands are transmitted without any modification and the system remains in the linear regime. Nevertheless, for this study, the deflection limit is reduced down to $\pm 0.8^{\circ}$. The deflection limit is normalized with respect to the reference command of $1.7^{\circ}$, which equals the flap command if no nonlinearities are active. The performance is hardly affected by this nonlinearity. Even if the deflection is limited to $60 \%$ of the reference flap command, the performance is still around $97 \%$ of the original moment reduction. Although the describing function of deflection limit indicates no phase variation, the phase increases with increasing deflection limit. However, it is noteworthy that the phase is not given
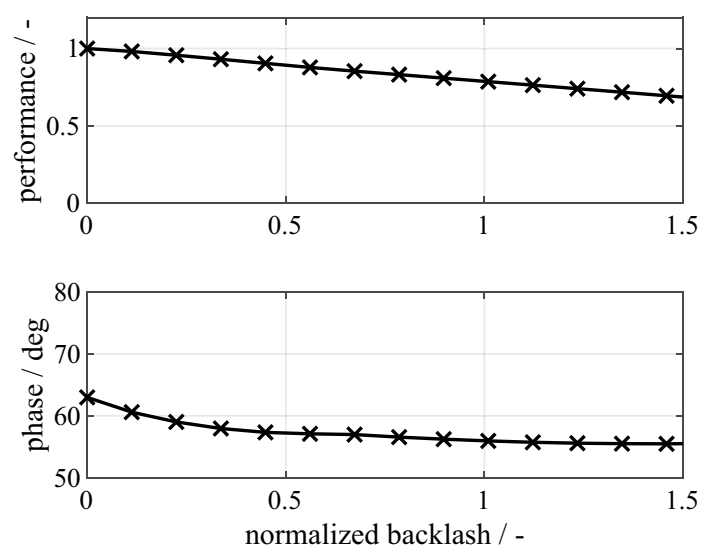

Fig. 9 Varying backlash and resulting performance loss of the controller from commanded deflection to actual flap deflection but between pitch excitation and wing root bending moment in Fig. 10. As a result, controller, actuators, structure as well as aerodynamics are affecting the change of phase as depicted in Fig. 10.

\subsubsection{Rate limit}

In a further study, the effect of rate limit is investigated. Again, the rate limit is normalized with respect to the maximum reference rate of $86.3 \mathrm{deg} / \mathrm{s}$ given by the controller in the linear case. Rate limits down to $60 \%$ of the maximum reference rate have no significant impact on the system. The performance is maintained at around $98 \%$ of the reference performance, see Fig. 11. Also, the phase lag is increasing with increasing rate limit.
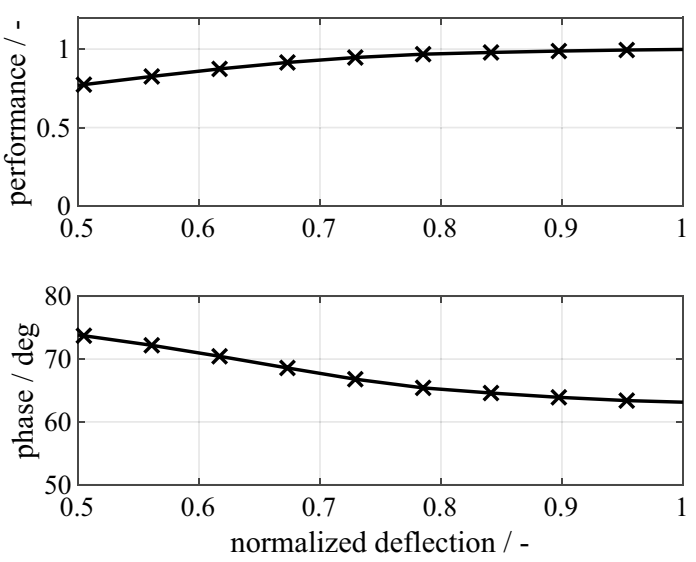

Fig. 10 Varying deflection limit and resulting performance loss of the controller
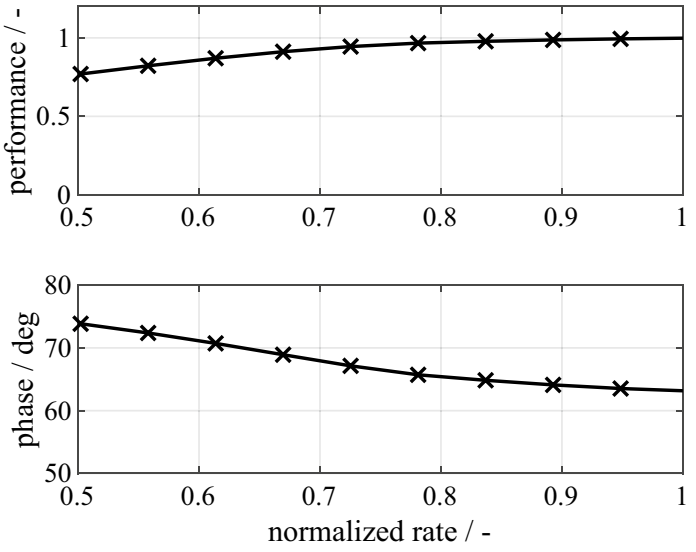

Fig. 11 Varying rate limit and resulting performance loss of the controller 

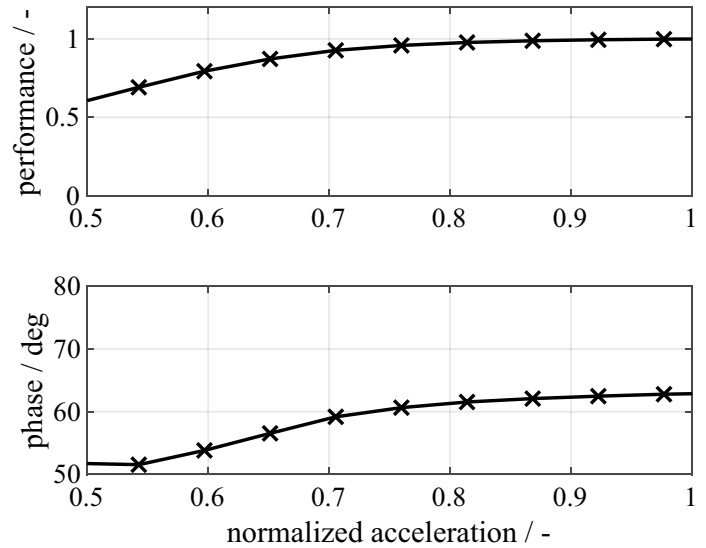

Fig. 12 Varying acceleration limit and resulting performance loss of the controller

\subsubsection{Acceleration limit}

Finally, the effect of acceleration limit is investigated. The given acceleration limit is normalized to the maximum reference acceleration of $4342 \mathrm{deg} / \mathrm{s}^{2}$. If the acceleration limit is around $60 \%$ of the linear response, the performance of the controller drops to $50 \%$ of the linear performance, as shown in Fig. 12. Thereby, the phase decreases with increasing acceleration limits. The nonlinearities due to rate limit and acceleration limit have a higher impact at higher frequencies. The rate increases with increasing frequency assuming constant amplitude while the acceleration increases by the power of 2. Consequently, the impact of the acceleration limit is greater than the rate limit.

\section{Experiment}

This section describes the testing of the servo. First, the design of the servo is presented and two servos are compared in a test bench. From which finally one servo is selected. Further testing is conducted to identify nonlinear parameters for this actuator. These parameters are then compared in a validation step with the theoretical model and performance loss is estimated from previous simulations.

\subsection{Actuator requirements}

In a first step of the design phase, the servo motor is selected. This component dominates the dynamic behavior of the whole system. Ravenscroft [11] describes which performance properties of an actuation system for a full scale aircraft are important for flight control. Although those actuation systems are generally driven by hydraulic systems, basic properties are the same. Stall load, roll-off frequencies and maximum rates exist for both actuator types. The stall load of the actuator is chosen such that the actuator can hold its position even when the highest aerodynamic load is applied. The rate capability is important for pilot handling. In this work, no handling qualities are considered, so no explicit rate is defined but implicitly in the required rolloff frequency for load alleviation. The bandwidth needed in this application is higher than the bandwidth for handling qualities. In contrast to flight control, the actuation system should be able to interact with the structural modes. This load alleviation system increases the damping of the first structural mode. As a rule of thumb, the bandwidth of the actuator is chosen at least at double the eigenfrequency of the structural mode.

The minimum actuator requirement for the considered wing is a bandwidth up to $16 \mathrm{~Hz}$ and a maximum deflection of $10^{\circ} .10^{\circ}$ includes a safety factor, since a smaller value around $2^{\circ}$ is adequate for the test. Rate limits should be as high as possible and backlash as small as possible. Maximum load on the actuator is predicted to be negligible.

\subsection{Actuation system design}

In this project, bought-in parts are used for a simpler design process. A direct drive at the hinge line was not possible due to limited space. A servo with drive mechanism was found to fit best for this project. The actuation system of the considered flexible wing consists of a flap, a servo and a mechanical drive mechanism. The drive mechanism has a transmission ratio of $1: 1$ and is built with a rod and a linkage to flap and servo respectively, as indicated in Fig. 13 top. The flap is mounted on the wing structure and is driven by the mechanism, where each connection is a bearing which potentially introduces backlash. Figure 13 bottom depicts the assembled
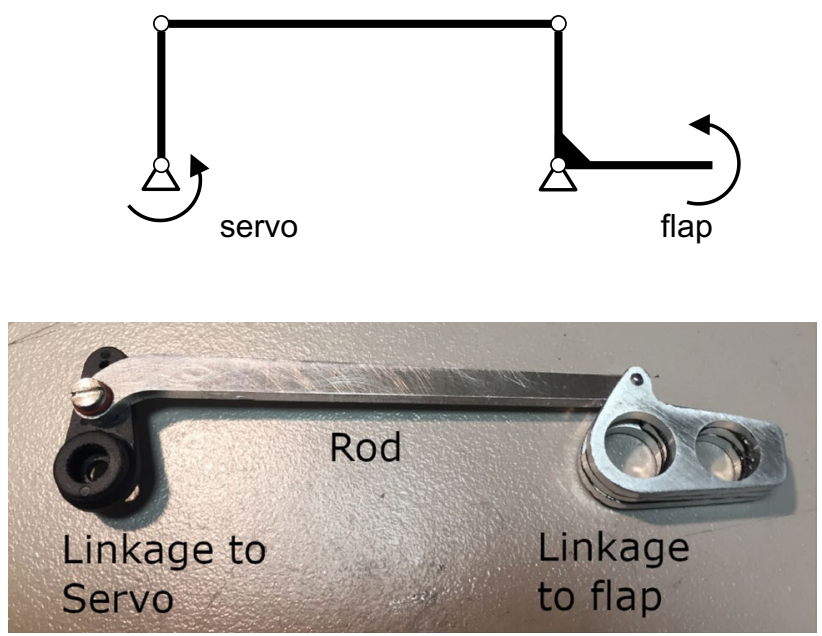

Fig. 13 Top: sketch of the mechanism of the actuator. Bottom: assembly of the mechanism 


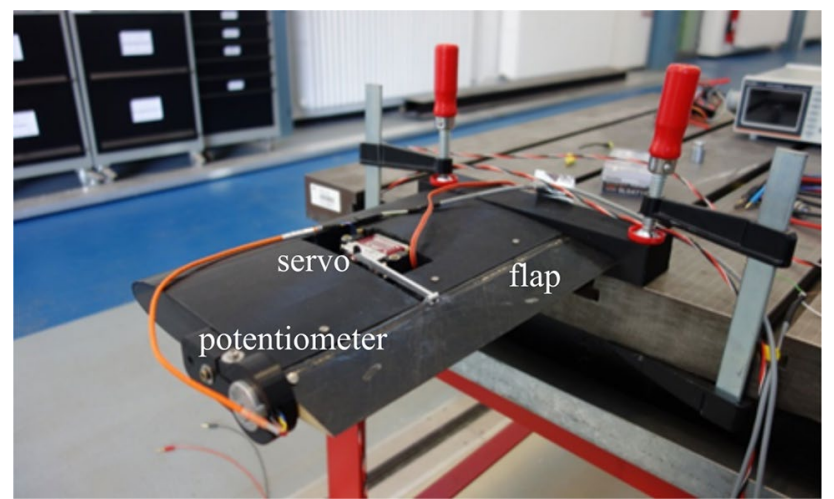

Fig. 14 Test bed for early integration test. First design of the actuator is tested

mechanism, which is also built into the test rig shown in Fig. 14.

\subsection{Test bed}

Similar to Regan [22], servo motor candidates were required to be tested before the wing model was built. To enable this, a test rig was designed to emulate the boundary condition of the wing, see Fig. 14. While the wing box consisted of a 3D printed mock-up, the flap in the test rig was supposed to be integrated in the final wing. Also, the mechanical drive mechanism between flap and servo represents the draft design for wing integration. The test served as a first integration test to check for geometric constraints and mechanical limits to avoid collisions. In the end, the mechanical design was slightly modified from the insights gained of this first integration test. For the experimental identification, a potentiometer is attached on the extension of the control surface hinge line to measure the rotary deflection of the flap directly.

First, the Futaba BLS471SV chosen by Regan [22] for a similar project is tested and second, the MKS motor is chosen as another option due to promising specifications given by the manufacturer. Dead time, rate limit, frequency response is assessed in the test bed and compared. In the end, MKS showed a better dynamic behavior. A comparison of the two frequency responses for 10deg deflection is shown in Fig. 15. The bandwidth of the MKS servo is higher and the gain around the rolloff frequency remains constant, whereas the gain of the Futaba increases. Also linear phase response is seen up to approximately $10 \mathrm{~Hz}$. More results on the dynamics of the MKS servo motor are presented in the next section.
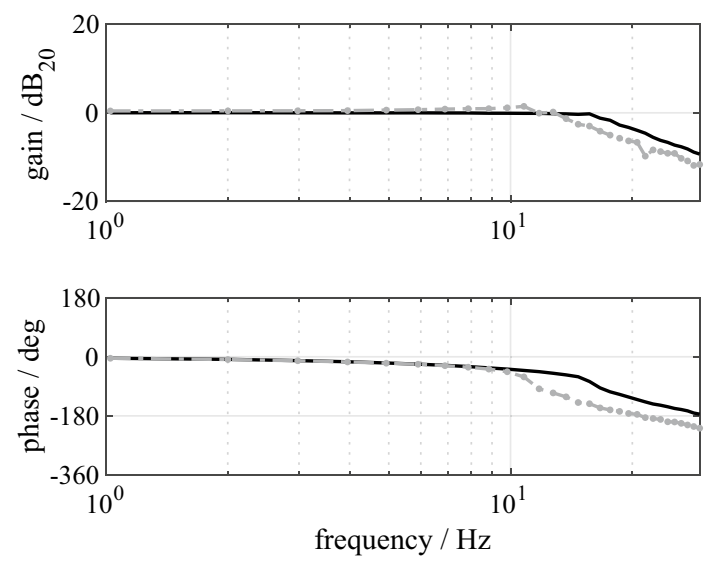

Fig. 15 Comparison of the two servos in the test bed. Black: MKS, gray: Futaba

\subsection{Actuator identification}

Finally, the parameters of the nonlinear system shown in Fig. 7 are identified. Often, non-parametric identification is performed in a first step before the system parameters are determined. A common approach to non-parametric identification of a dynamic system is to excite the system with a known input signal and observe the output response. Depending on the intended usage of the identified model, typical excitation signals include step function, random or sine sweep. For example, the sine sweep concentrates the excitation energy on a narrow band sliding through the frequency range of interest, whereas with random excitation the excitation energy is spread over a broad frequency band. Thus, the sine sweep leads to higher amplitudes and quasiharmonic response which is preferable for nonlinear identification. Transfer functions are used to describe dynamic systems in frequency domain without parameters. For this, the input signal $u(t)$ and output signal $y(t)$ are measured first. Next, both signals are transformed to frequency domain with a Fourier transform. Finally, the transfer function $H(\omega)=\frac{Y(\omega)}{U(\omega)}$ is given as ratio between input and output.

Note that this assumes linear dynamics, since the transfer function for nonlinear systems is actually not existent. In a first approach, however, the system is assumed to be linear and is identified according to this relation. The response at different amplitude levels also reveals some information on the existence and type of nonlinearity. For example, a linear phase loss is introduced by dead time, which can be directly identified by determining the linear slope of the phase response.

The transfer behavior of the servo is identified at first in a non-parametric way using input-output transfer functions. Afterwards, dedicated test signals, following the methodology from Regan [22], are used to experimentally identify 


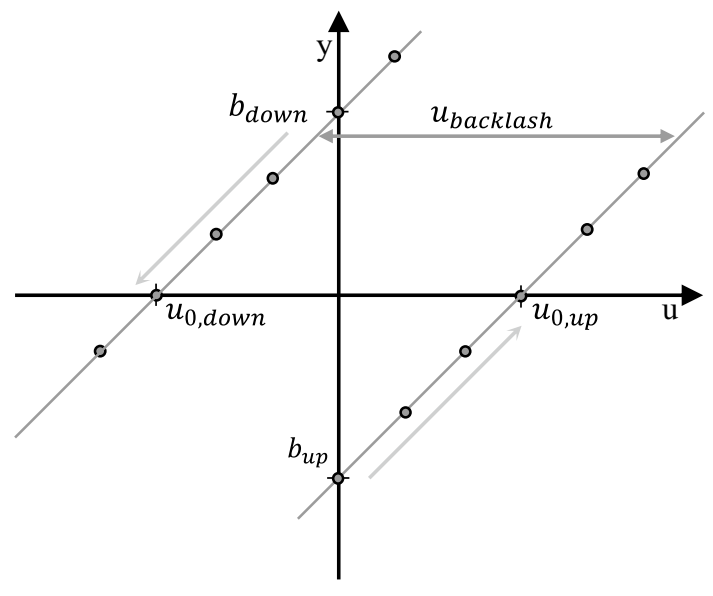

Fig. 16 Sketch of measured up and down path for free play identification

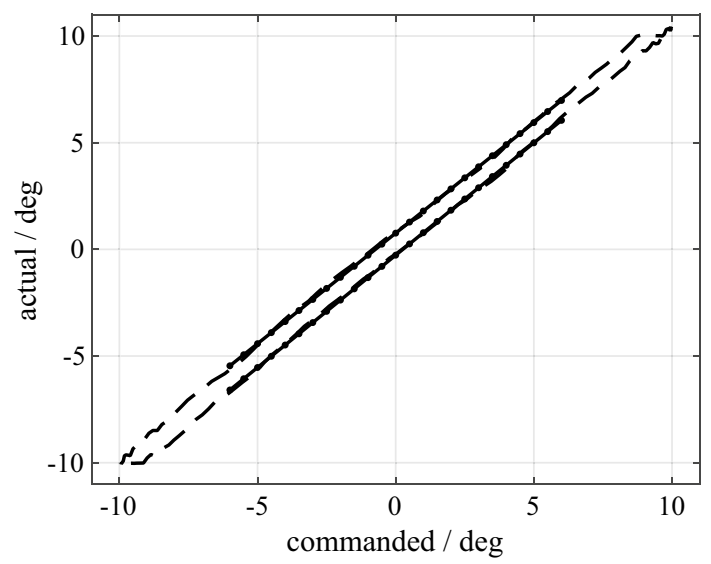

Fig. 17 The dashed line depicts the actual deflection over commanded deflection, hysteresis due to backlash is clearly visible. The solid line depicts a linear regression for up and down movement. The offset equals backlash

specific parameters of different nonlinearities involved in the actuator model. The maximum achievable rate is identified from step function inputs and the backlash parameter is quantified from sine excitation at a low frequency. The transfer functions measured at different amplitude levels reveal the acceleration limit of the actuator. With the identified parameters, a nonlinear Simulink model for the actuator is established.

\subsubsection{Backlash}

A very-low-frequency sine excitation is used to identify the backlash in the mechanical actuation of the flap. Figure 17 shows the measured commanded flap signal and actual flap deflection signal, measured by the potentiometer in the flap hinge. The plot of the actual flap signal over the commanded flap signal forms a hysteresis, which is typical for backlash. At the turning points (i.e., upper right hand corner and lower left hand corner of the hysteresis), the flap is actually not moving even though the commanded flap signal changes. When the flap is out of the backlash area, the actual flap signal starts following the commanded flap signal. As a consequence, the backlash corresponds to the width of the hysteresis, as sketched out in Fig. 16.

The hysteresis has an upward path and a downward part. Both are linear functions with slope $a$ and offset $b$ which are identified separately for each path. To distinguish the data samples in the upward path from those in the downward path, the difference between two subsequent samples in the commanded signal is used. If the difference is positive, the sample belongs to the upward path, if it is negative, the sample belongs to the downward path. Now, the slope $a$ and the offset $b$ can be identified once for the upward path and once for the downward path. This is done in a range from $-5^{\circ}$ to $5^{\circ}$ commanded flap deflection.

The identification of the slope $a$ and the offset $b$ is performed by curve fitting. This is essentially a regression, in which more data samples are employed as parameters to be identified. This ensures adequate accuracy, independence from random measurement errors and robust parameter estimates.

The model equation used for curve-fitting the upward path and the downward path is shown in Eq. (3):

$y_{\mathrm{i}}=a u_{\mathrm{i}}+b$,

where $y_{i}$ is the sample of the actual flap signal, $u_{i}$ is the sample of the commanded flap signal at time $t_{i}, a$ is the slope, and $b$ is the offset. This equation can be employed for $i=1,2, \ldots n$ data samples, so that Eq. (3) is developed into an overdetermined equation system, which can be solved in least-squares sense to obtain the parameters $a$ and $b$ :

$\left\{\begin{array}{c}y_{1} \\ \vdots \\ y_{\mathrm{n}}\end{array}\right\}=\left[\begin{array}{cc}u_{1} & 1 \\ \vdots & \vdots \\ u_{\mathrm{n}} & 1\end{array}\right]\left\{\begin{array}{l}a \\ b\end{array}\right\}$,

$\{y\}=[A]\{p\}$,

$\{p\}=\left([A]^{T}[A]\right)^{-1}[A]^{T}\{y\}$.

This curve fitting is performed on the upward path and the downward path separately yielding the parameters $a_{\text {up }}$ and $b_{\text {up }}$ and $a_{\text {down }}$ and $b_{\text {down }}$ respectively, as indicated in Fig. 16. The slope $a_{\text {up }}$ equals the slope $a_{\text {down }}$ as can be seen in measured hysteresis in Fig. 17. With the parameters $a$ and $b$ for the upward and downward path, the zero points are obtained with $u_{0}=-\frac{a}{b}$. The backlash $u_{\text {backlash }}$ is now computed as 


$$
u_{\text {backlash }}=u_{0, \text { up }}-u_{0, \text { down }}=\frac{a_{\text {down }}}{b_{\text {down }}}-\frac{a_{\text {up }}}{b_{\text {up }}} .
$$

\subsubsection{Rate limit}

The rate limit of the actuator is tested using step functions of different step levels. The slope of the response is the rate limit. Figure 18 depicts the measured step responses. To estimate the slope or also maximum rate, only the linearly increasing part is considered. With this data, a linear regression is applied to find the slope. Since several data points are incorporated, robustness against measurement noise is increased. Again, Eq. (3) is applied, where in this case $u$ corresponds to time and $y$ to the actual deflection. The slope $a$ corresponds to the estimated velocity limit.

The dashed line shows the response for a $7^{\circ}$ step command signal and the dotted line represents the response of the actuator for a $57^{\circ}$ step signal. The circles are fitted to the $57^{\circ}$ step and the diamonds are fitted to the $7^{\circ}$ step. The estimated slope for the response of the $7^{\circ}$ step reveals a maximum rate of $339 \mathrm{deg} / \mathrm{s}$, whereas the linear fit of the response of the $57^{\circ}$ step shows a maximum velocity of $1129 \mathrm{deg} / \mathrm{s}$.

It seems that the maximum velocity increases with the step command. It is assumed that the torque limit might be responsible for this. There is simply not enough time for the actuator to reach the maximum velocity at smaller step commands. Also, the unknown dynamics of the internal tracking controller might be a reason for this behavior. However, if the step is high enough, it is supposed that the flap has enough time to

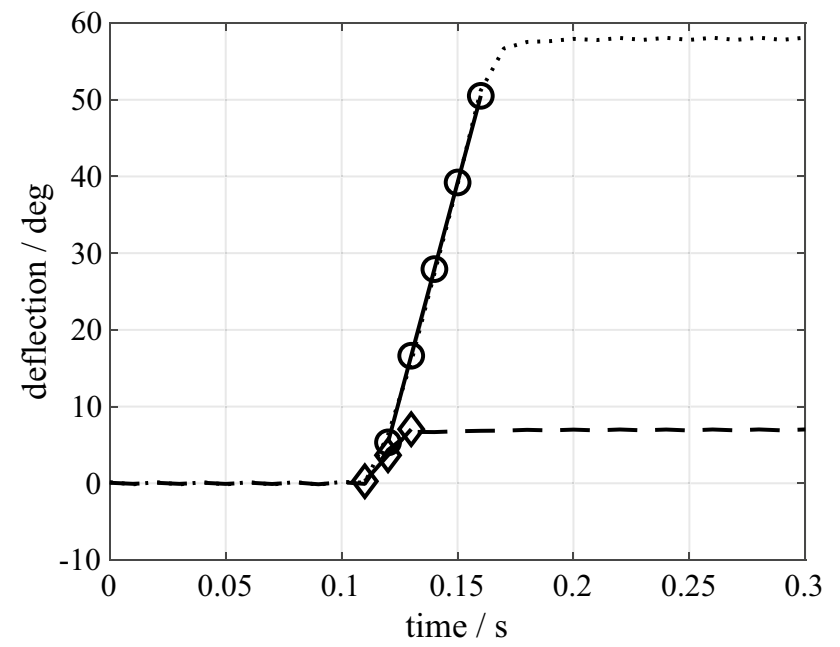

Fig. 18 Estimation of the rate limit using a step function. Dashed: $7^{\circ}$ step response, dotted: $57^{\circ}$ step response, diamonds: identified slope of $339 \mathrm{deg} / \mathrm{s}$ for $7^{\circ}$ step, circles: identified slope of $1129 \mathrm{deg} / \mathrm{s}$ for $57^{\circ}$ step accelerate to its true rate limit. Hence, $1129 \mathrm{deg} / \mathrm{s}$ is identified as rate limit for this actuator.

\subsubsection{Roll-off frequency and dead time}

To identify the dynamics for different amplitudes, sweep excitations are used. The results are shown in Fig. 19 as deflection amplitude over frequency. From this, a linear model can be derived for each amplitude level. But it can be clearly seen (e.g., solid curve in Fig. 19) that the actuator runs into some kind of saturation at higher frequencies. Hence, a nonlinear model including rate and power limits is considered. The socalled underlying linear model is identified from the low level run at $3^{\circ}$. A model of first order is assumed to be sufficient to represent the linear dynamic behavior. For a first-order system, one parameter is feasible to fully determine the dynamics, namely the roll-off frequency. The transfer function $H$ is given as $H(\omega)=\frac{K}{j \omega T+1}$. Since the phase response is affected by dead time but not the amplitude response, only the amplitude response is used for roll-off frequency identification. For the identification of the roll-off frequency $T^{-1}$ and the gain $K$, the corresponding model equation is developed from the analytical transfer function of a first-order system. Rearranging the transfer function results in

$H(\omega)=K-j \omega T H(\omega)$.

The transfer function $H$ is measured with discrete number of excitation frequencies $\omega_{i}$, with $i=1,2, \ldots, n$. With $H_{i}=H(\omega)$, the following model equation is derived:
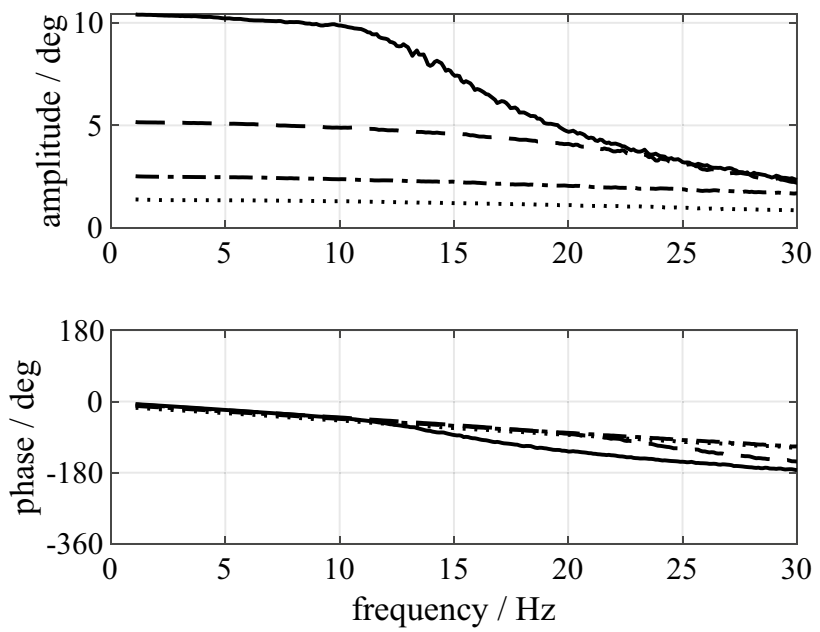

Fig. 19 Frequency responses for different excitation level. Dotted: $3^{\circ}$, dash-dotted: $5^{\circ}$, dashed: $10^{\circ}$, solid: $20^{\circ}$ 


$$
\left\{\begin{array}{c}
H_{1} \\
\vdots \\
H_{\mathrm{n}}
\end{array}\right\}=\left[\begin{array}{cc}
1 & -j \omega H_{1} \\
\vdots & \vdots \\
1 & -j \omega H_{n}
\end{array}\right]\left\{\begin{array}{c}
K \\
T
\end{array}\right\}=[A]\{p\} .
$$

As dead time introduces an additional phase loss, magnitude only of the transfer function is considered. This is solved as least squares problem as in Eq. (5).

Also, a linear phase loss is seen, indicating a dead time, which is identified from the linear slope of the phase response. Only the region where phase loss is linear is chosen for the linear regression. This identification procedure is expressed in Eq. (3), where $a$ is the delay $\tau$ and $b$ is incorporated to consider bias of the phase, possibly introduced by backlash.

\subsubsection{Acceleration limit}

The measured amplitude responses are differentiated in frequency domain to study the acceleration amplitude over frequency, see Fig. 20. As one can see, the acceleration cannot exceed a certain value, which is the actual acceleration limit. This value is computed as average of the constant part. This limitation depends on the actual torque limit of the actuator as well as on the inertia of the connected flap.

\subsection{Validation of identified parameters}

The identified parameters are shown in Table 1 and used to build a nonlinear simulation model of the actuator as described in Sect. 2.3.3. Figure 21 shows simulated transfer functions with the presented identified parameters and the measured transfer function. Generally, good agreement is seen. Note that the match improves with higher amplitudes.
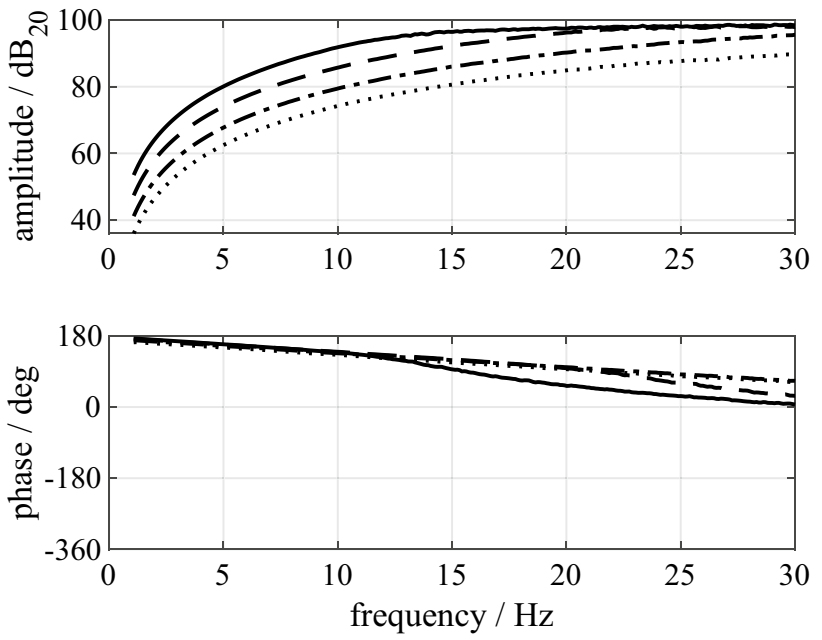

Fig. 20 Acceleration response for different excitation level. Dotted: $3^{\circ}$, dash-dotted: $5^{\circ}$, dashed: $10^{\circ}$, solid: $20^{\circ}$
Table 1 Identified parameters of the actuator

\begin{tabular}{lll}
\hline Parameter & Value & Normalized \\
\hline Roll of frequency & $25 \mathrm{~Hz}$ & - \\
Dead time & $4.3 \mathrm{~ms}$ & - \\
Backlash & $1^{\circ}$ & 0.59 \\
Deflection limit & $10^{\circ}$ & 4.7 \\
Rate limit & $1129 \mathrm{deg} / \mathrm{s}$ & 12 \\
Acceleration limit & $79,540 \mathrm{deg} / \mathrm{s}^{2}(98 \mathrm{~dB})$ & 18 \\
\hline
\end{tabular}

As already discussed, backlash introduces a constant offset in phase and gain varying with amplitude. This effect is reduced for higher amplitudes of commanded deflection as it can be seen in Fig. 21. Another nonlinear effect is the amplitude dependent roll-off introduced by the acceleration limit.

Table 1 also includes normalized values with the reference values from the simulation section. Only backlash will have an impact on controller performance. All other values are high enough, that the actuator will not run into limits. This means that Fig. 9 at normalized backlash of 0.59 is the predicted performance for this actuator. With this relatively high backlash, around $90^{\circ}$ of the nominal performance can be achieved.

\section{Conclusion}

A parametric nonlinear model of a servo for the actuation of a control surface is derived and implemented in Simulink. The nonlinear actuator model is then integrated in an aeroservoelastic simulation model, including DLM-based unsteady aerodynamics and linear structural dynamics. The impact of the nonlinear parameters for backlash and limits in deflection, rate and acceleration on the load alleviation
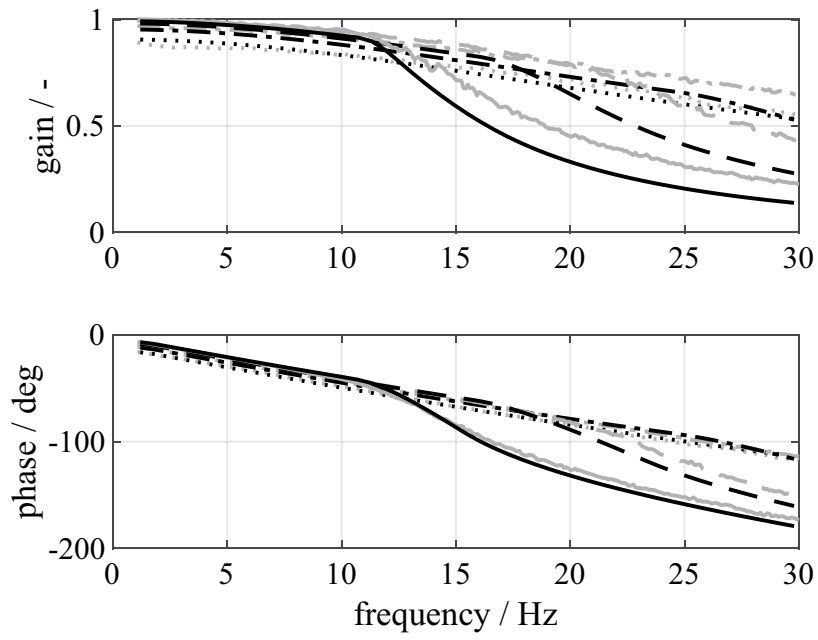

Fig. 21 Comparison of measured and simulated actuator dynamics. Black lines are measured and gray lines are simulated dynamics. Dotted: $3^{\circ}$, dash-dotted: $5^{\circ}$, dashed: $10^{\circ}$, solid: $20^{\circ}$ 
effectiveness are investigated in simulations. Backlash and acceleration limit are found to have the highest impact on the closed-loop performance. Due to limits in deflection, rate and acceleration and also due to backlash, actual flap deflections are always smaller than the commanded flap deflection. Hence, the desired reduction in wing root bending moment is overestimated in numerical simulations conducted with a linear equivalent model of the actuator. However, the controller designed for the linear system partially compensates for this and amplifies the deflection commands such that the measured wing root bending moment is further decreased. However, due to the phase lag introduced by the backlash, the controller is not always able to fully compensate the nonlinear effects. Finally, the parameters for the nonlinearities in the actuation system are identified for a given servo, used for the actuation in a flexible wind tunnel model. Different input signals are utilized to identify Parameters of limits and backlash. The test bed is presented with the basic procedure applied to identify the parameter values. Finally, it should be mentioned that the methodology presented here can be applied systematically in numerical investigations of actuator nonlinearities on the performance of load alleviation systems.

Acknowledgements The authors would like to thank Mr. Tobias Meier for his valuable support in building up the testbed for actuator identification. This work was commonly conducted by the DLR-Institute of Aeroelasticity and DLR-Institute of System Dynamics and Control within the DLR-internal Aeronautical Research Project "KonTeKst".

Funding Open Access funding enabled and organized by Projekt DEAL.

Open Access This article is licensed under a Creative Commons Attribution 4.0 International License, which permits use, sharing, adaptation, distribution and reproduction in any medium or format, as long as you give appropriate credit to the original author(s) and the source, provide a link to the Creative Commons licence, and indicate if changes were made. The images or other third party material in this article are included in the article's Creative Commons licence, unless indicated otherwise in a credit line to the material. If material is not included in the article's Creative Commons licence and your intended use is not permitted by statutory regulation or exceeds the permitted use, you will need to obtain permission directly from the copyright holder. To view a copy of this licence, visit http://creativecommons.org/licenses/by/4.0/.

\section{References}

1. Ossmann, D., Pusch, M.: Fault tolerant control of an experimental flexible wing. Aerospace 6(7), 76 (2019)

2. Dillinger, J., Meddaikar, M.Y., Lübker, J., Pusch, M., Kier, T.: Design and optimization of an aeroservoelastic wind tunnel model. In: International Forum on Aeroelasticity and Structural Dynamics (2019)

3. Krüger, W.R., Dillinger, J., Meddaikar, M.Y., Lübker, J., Tang, M., Meier, T., Böswald, M., Soal, K.I., Pusch, M., Kier, T.: Design and wind tunnel test of an actively controlled flexible wing. In: International Forum on Aeroelasticity and Structural Dynamics (2019)

4. Klimmek, T., Bogenfeld, R., Breitbarth, E., Dillinger, J., Handojo, V., Kier T., Kohlgrüber, D, Pusch, M., Raab, C., Wild, J.: Aircraft loads - a wide range of disciplinary and process-related issues in simulation and experiment. In: Deutscher Luft- und Raumfahrt Kongress, vol. 9 (2020)

5. Fung, Y.-C.: An Introduction to the Theory of Aeroelasticity. Wiley, New York (1955)

6. Fielding, C., Flux, P.K.: Non-linearities in flight control systems. Aeron. J. 107(1077), 673-686 (2003)

7. Theis, J., Pfifer, H., Seiler, P.J.: Robust control design for active flutter suppression. In: AIAA Atmospheric Flight Mechanics Conference, p. 1751 (2016)

8. Pusch, M., Ossmann, D., Luspay, T.: Structured control design for a highly flexible flutter demonstrator (2019)

9. Giurgiutiu, V.: Review of smart-materials actuation solutions for aeroelastic and vibration control. J. Intell. Mater. Syst. Struct. 11(7), 525-544 (2000)

10. Fu, J., Mare, J.C., Fu, Y.L.: Modelling and simulation of flight control electromechanical actuators with special focus on model architecting, multidisciplinary effects and power flows. Chin. J. Aeron. 30(1), 47-65 (2017)

11. Taylor, R., Pratt, R.W., Caldwell, B.D.J.: The application of actuator performance limits to aeroservoelastic compensation. Trans. Inst. Meas. Control 21(2-3), 106-112 (1999)

12. Stirling, R., Cowling, D.: Implementation of comprehensive actuation system models in aeroservoelastic analysis. In: European Forum on Aeroservoelasticity and Structural Dynamics, Aachen, Germany, report no. SDL, vol. 143

13. Banavara, N.K., Newsom, J.R.: Framework for aeroservoelastic analyses involving nonlinear actuators. J. Aircr 49(3), 774-780 (2012)

14. Klyde, D.H., Mitchell, D.G.: Investigating the role of rate limiting in pilot-induced oscillations. J. Guid. Control Dyn. 27(5), 804-813 (2004)

15. Silvestre, F.J., Guimarães Neto, A.B., Bertolin, R.M., da Silva, R.G.A., Paglione, P.: Aircraft control based on flexible aircraft dynamics. J. Aircr. 54(1), 262-271 (2017)

16. Theis, J., Pfifer, H., Balas, G., Werner, H.: Integrated flight control design for a large flexible aircraft. In: 2015 American Control Conference (ACC), Chicago, IL, pp. 3830-3835 (2015)

17. Schmidt, D.: Modern Flight Dynamics. McGraw-Hill, New York (2012)

18. Singh, K.V., Brown, R.N., Kolonay, R.: Receptance-based active aeroelastic control with embedded control surfaces having actuator dynamics. J. Aircr. 53(3), 830-845 (2016)

19. Schallert, C., Kowalski, R., Rottach, M., Dorkel, A.: Automated measurment of backlash and stiffness in electro-mechanical flight control actuation. In: Recent Advances in Aerospace Actuation Systems and Components, pp. 103-108 (2018)

20. Cologni, A.L., Mazzoleni, M., Previdi, F.: Modeling and identification of an electro-hydraulic actuator. In: Proceedings of the 30th IEEE Conference on Control and Automation, pp. 335-340 (2016)

21. Ling, T.G., Rahmat, M.F., Husain, A.R., Ghazali, R.: System identification of electro-hydraulic actuator servo system. In: Proceedings of the 4th International Conference on Mechatronics, pp. 1-7 (2011)

22. Regan, C: maewing2: conceptual design and system test. In: AIAA Atmospheric Flight Mechanics Conference (2017)

23. Danowski, B., Thompson, P.M., Kukreja, S.: Nonlinear analysis of aeroservoelastic models with free play using describing functions. J. Aircr. 50(2), 329-336 (2013) 
24. Ackermann, J., Bünte, T.: Robust prevention of limit cycles for robustly decoupled car steering dynamics. Kybernetika 35(1), 105-116 (1999)

25. Tang,M., Böswald, M., Govers, Y., Pusch, M.: Identification and assessment of a nonlinear dynamic actuator model for gust load alleviation in a wind tunnel experiment. In: Deutscher Luft- und Raumfahrtkongress (2019)

26. Pusch, M., Ossmann, D., Kier, T., Dillinger, J., Tang, M., Lübker, J.: Aeroelastic modeling and control of an experimental flexible wing. In: AIAA SciTech Forum (2019)

27. Dillinger, J.K.S., Klimmek, T., Abdalla, M.M., Gürdal, Z.: Stiffness optimization of composite wings with aeroelastic constraints. J. Aircr 50(4), 1159-1168 (2013)

28. Dillinger, J.K.S., Meddaikar, Y.M., Lübker, J., Pusch, M., Kier, T.: Design and optimization of an aeroservoelastic wind tunnel model. Fluids 5, 35 (2020). https://doi.org/10.3390/fluids5010035

29. Meddaikar, M.Y., Dillinger, J., Ritter, M.R., Govers, Y.: Optimization \& testing of aeroelastically-tailored forward swept wings. In: International Forum on Aeroelasticity and Structural Dynamics (2017)
30. Rodden, W.P., Giesing, J.P., Kalman, T.P.: Refinement of the nonplanar aspects of the subsonic doublet-lattice lifting surface method. J. Aircr 9(1), 69-73 (1972)

31. Roger, K.L.: Airplane math modeling methods for active control design (1977)

32. Varga, A. Balancing-free square-root algorithm for computing singular perturbation approximations. In: Proceedings of the 30th IEEE Conference on Decision and Control, pp. 1062-1065 (1991)

33. Kier, T., Looye, G.: Unifying manoeuvre and gust loads analysis. In: International Forum on Aeroelasticity and Structural Dynamics (2009)

34. Pusch, M.: Aeroelastic mode control using $H_{2}$-optimal blends for inputs and outputs. In: AIAA Guidance, Navigation, and Control Conference (2018)

Publisher's Note Springer Nature remains neutral with regard to jurisdictional claims in published maps and institutional affiliations. 